CLINICAL STUDY

\title{
Epidemiology of adrenal crisis in chronic adrenal insufficiency: the need for new prevention strategies
}

\author{
Stefanie Hahner ${ }^{1}$, Melanie Loeffler ${ }^{1}$, Benjamin Bleicken ${ }^{3}$, Christiane Drechsler ${ }^{2}$, Danijela Milovanovic ${ }^{1}$, \\ Martin Fassnacht ${ }^{1}$, Manfred Ventz ${ }^{3}$, Marcus Quinkler ${ }^{3}$ and Bruno Allolio ${ }^{1}$ \\ ${ }^{1}$ Endocrinology and Diabetes Unit, Department of Medicine I, ${ }^{2}$ Department of Nephrology, Department of Medicine I, University of Würzburg, \\ Oberdürrbacher Str. 6, D-97080 Würzburg, Germany and ${ }^{3}$ Clinical Endocrinology, Department of Medicine, Gastroenterology, Hepatology and \\ Endocrinology, Charité University Medicine Berlin, Charité Campus Mitte, 10117 Berlin, Germany \\ (Correspondence should be addressed to B Allolio; Email: allolio_b@medizin.uni-wuerzburg.de)
}

\begin{abstract}
Objective: Adrenal crisis (AC) is a life-threatening complication of adrenal insufficiency (AI). Here, we evaluated frequency, causes and risk factors of AC in patients with chronic AI.

Methods: In a cross-sectional study, 883 patients with AI were contacted by mail. Five-hundred and twenty-six patients agreed to participate and received a disease-specific questionnaire.

Results: Four-hundred and forty-four datasets were available for analysis (primary AI (PAI), $n=254$; secondary AI (SAI), $n=190$ ). Forty-two percent (PAI 47\% and SAI 35\%) reported at least one crisis. Three hundred and eighty-four AC in 6092 patient years were documented (frequency of 6.3 crises/100 patient years). Precipitating causes were mainly gastrointestinal infection and fever (45\%) but also other stressful events (e.g. major pain, surgery, psychic distress, heat and pregnancy). Sudden onset of apparently unexplained AC was also reported (PAI 6.6\% and SAI 12.7\%). Patients with PAI reported more frequent emergency glucocorticoid administration $(42.5$ vs $28.4 \%, P=0.003)$. Crisis incidence was not influenced by educational status, body mass index, glucocorticoid dose, DHEA treatment, age at diagnosis, hypogonadism, hypothyroidism or GH deficiency. In PAI, patients with concomitant non-endocrine disease were at higher risk of crisis (odds ratio $(\mathrm{OR})=2.02,95 \%$ confidence interval $(\mathrm{CI}) 1.05-3.89, P=0.036)$. In SAI, female sex $(\mathrm{OR}=2.18,95 \% \mathrm{CI} 1.06-4.5$, $P=0.035)$ and diabetes insipidus $(\mathrm{OR}=2.71,95 \% \mathrm{CI} 1.22-5.99, P=0.014)$ were associated with higher crisis incidence.

Conclusion: AC occurs in a substantial proportion of patients with chronic AI, mainly triggered by infectious disease. Only a limited number of risk factors suitable for targeting prevention of AC were identified. These findings indicate the need for new concepts of crisis prevention in patients with AI.
\end{abstract}

European Journal of Endocrinology 162 597-602

\section{Introduction}

Adrenal crisis (AC) is a life-threatening complication in patients suffering from chronic adrenal insufficiency (AI). It is well known that endogenous cortisol secretion increases substantially in response to stressful events in healthy subjects. In contrast, patients with AI need to adequately adjust their glucocorticoid dose in case of stress to cover the increased demand in adrenal steroids and to avert AC. However, the clinical features of impending AC are often non-specific, leading to delay of emergency glucocorticoid administration. Therefore, effective prevention of $\mathrm{AC}$ remains a major challenge in the care of patients with chronic AI.

Recent data demonstrate that mortality is increased in chronic AI (1-3). In an analysis of 1675 patients with Addison's disease from the National Swedish Hospital and Cause of Death Registry, 507 deaths were documented compared to an expected number of only 199 deaths. Sixty-four (12.6\%) of these deaths were attributed to 'endocrine causes' with 36 cases (7.1\%) identified as death from AI per se (1). This suggests that AC substantially contributes to the increased mortality in AI.

To date, only limited data are available regarding incidence and precipitating causes of $\mathrm{AC}$ mainly consisting of single case reports. In undiagnosed AI, initiation of thyroxine replacement may induce AC due to increased cortisol metabolism (4-6). Adrenostatic drugs like etomidate or ketoconazole or drugs that accelerate cortisol metabolism like barbiturates, rifampicin or mitotane may trigger $\mathrm{AC}(7-9)$. In a small survey in patients with Addison's disease, 11 of 25 patients $(44 \%)$ had a history of AC requiring inpatient treatment with i.v. hydrocortisone administration (10). Nagesser et al. (11) reported Addisonian crisis requiring hospitalization and treatment with i.v. fluids and corticosteroids in 9 of 44 patients $(20 \%)$ 
after bilateral adrenalectomy for Cushing's disease. Thompson et al. also assessed 39 patients after bilateral adrenalectomy for Cushing's disease with a mean duration after adrenalectomy of 3.6 years (12). In their analysis, 9 of 39 (23\%) patients experienced an AC leading to hospital admission. In the largest so far retrospective analysis of medical records of 137 patients with primary and secondary AI (PAI, SAI), Omori et al. found that $29 \%$ of these patients had experienced at least one AC (13). In their analysis, mental disorder and untreated hypogonadism were associated with the occurrence of AC.

To protect patients from life-threatening AC, patient education is considered the most important preventive measure (14). However, efficacy of patient education has never been evaluated. Obviously, defining patients at high risk for $\mathrm{AC}$ would be helpful to better target specific preventive measures. Thus, we performed a postal survey in a large cohort of patients with either primary or SAI to further assess the incidence and precipitating causes of $\mathrm{AC}$ and to identify potential risk factors that may help to improve patient surveillance and education.

\section{Subjects and methods}

\section{Patients}

A subgroup of our patient cohort has previously been described in detail $(15,16)$. All patients with AI currently registered with the outpatient department at the University Hospital Würzburg $(n=148)$ and the University Hospital Charité Berlin $(n=535)$, and registered members of the German self-help network of patients with pituitary or adrenal diseases suffering from AI $(n=200)$ were asked to participate in a postal survey. The study was approved by the ethical committee of the Universities of Würzburg (permit no. 45/04) and Berlin (permit no. ES1/037/06), and written informed consent was obtained from all patients prior to participation.

The underlying diagnosis of AI was verified by review of the medical records. In addition, the following exclusion criteria were applied: AI due to previous long-term pharmacological glucocorticoid treatment; glucocorticoid doses above $7.5 \mathrm{mg}$ prednisolone equivalent for other reasons than $\mathrm{AI}$; adrenocortical carcinoma; adrenoleukodystrophy; and patients with $<12$ months duration of disease.

\section{Questionnaires}

Patients willing to participate received a questionnaire that collected data on duration and cause of $\mathrm{AI}$, hormone replacement therapy, further medication, additional endocrine or general health problems, educational status, occupational status and the self-perceived influence of AI on activities of daily life.
AC was defined as acute impairment of general health with need of i.v. glucocorticoid administration and requiring hospital admission. Patients were further asked for the need of emergency i.v. glucocorticoid administration, frequency and causes of AC, equipment with an emergency card and provision of an emergency glucocorticoid kit. For every question, patients had to choose from a list of potential answers that were given with tick boxes. In addition, patients had the possibility to add further answers as free text.

\section{Statistical analysis}

Patients were divided into two groups according to the presence of primary or SAI, and stratified by sex. Continuous variables were expressed as median with interquartile range, as appropriate, and categorical variables were expressed as percentages. Comparisons across groups were calculated by Mann-Whitney $U$ test for continuous variables, and by $\chi^{2}$-tests for categorical variables.

The following analyses were performed in detail. First, the self-reported occurrence of AC was determined as the number of events per patient group, and as

Table 1 Age, duration of disease and underlying diagnosis in 444 patients with adrenal insufficiency.

\begin{tabular}{|c|c|c|c|}
\hline Primary adrenal insufficiency & \multicolumn{2}{|c|}{$\begin{array}{l}\text { Men } \\
(n=63)\end{array}$} & $\begin{array}{l}\text { Women } \\
(n=191)\end{array}$ \\
\hline \multicolumn{4}{|l|}{ Age (years) } \\
\hline Median (range) & \multicolumn{2}{|c|}{$46(20-84)$} & $46(20-80)$ \\
\hline \multicolumn{4}{|l|}{ Duration of disease (years) } \\
\hline Median (range) & \multicolumn{2}{|c|}{$10(1-50)$} & $12(1-45)$ \\
\hline \multicolumn{4}{|l|}{ Cause of adrenal insufficiency $(n)$} \\
\hline Autoimmune adrenalitis & \multicolumn{2}{|l|}{25} & 140 \\
\hline Bilateral adrenalectomy & \multicolumn{2}{|l|}{13} & 29 \\
\hline $\mathrm{CAH}$ & \multicolumn{2}{|l|}{8} & 18 \\
\hline Tuberculosis & \multicolumn{2}{|l|}{3} & 3 \\
\hline Unknown & \multicolumn{2}{|l|}{14} & 1 \\
\hline $\begin{array}{l}\text { Secondary adrenal } \\
\text { insufficiency }\end{array}$ & & $\begin{array}{l}\text { Men } \\
(n=77)\end{array}$ & $\begin{array}{l}\text { Women } \\
(n=113)\end{array}$ \\
\hline \multicolumn{4}{|l|}{ Age (years) } \\
\hline Median (range) & & $59(23-78)$ & $54(17-81)$ \\
\hline \multicolumn{4}{|l|}{ Duration of disease (years) } \\
\hline Median (range) & & $10(1-41)$ & $10(1-57)$ \\
\hline \multicolumn{4}{|l|}{ Cause of adrenal insufficiency $(n)$} \\
\hline Pituitary adenoma & & 46 & 61 \\
\hline Other intracerebral tumours ${ }^{a}$ & & 10 & 20 \\
\hline Lymphocytic hypophysitis & & 0 & 1 \\
\hline Sarcoidosis & & 1 & 1 \\
\hline Isolated ACTH deficiency & & 2 & 1 \\
\hline Aneurysm of the ant. a. communica & ans & 1 & 0 \\
\hline Empty sella & & 1 & 2 \\
\hline Brain injury & & 1 & 1 \\
\hline Sheehan's syndrome & & - & 12 \\
\hline Congenital dysplasia of pituitary & & 0 & 1 \\
\hline Unknown & & 15 & 13 \\
\hline
\end{tabular}

$\mathrm{CAH}$, congenital adrenal hyperplasia.

ather intracerebral tumours: craniopharyngioma $(n=22)$; sella turcica meningioma $(n=2)$; cholesterol granuloma $(n=1)$; dermoid cyst $(n=1)$; opticus glioma $(n=1)$; cyst at pituitary $(n=3)$. 
incidence rate with the number of events per 100 patient years in each group. Secondly, we determined the proportions of known factors preceding AC, as reported by the patients. Thirdly, we assessed the effects of potential risk factors on the occurrence of AC by binary logistic regression analyses. Odds ratios (OR) with 95\% confidence intervals (CIs) were calculated for the whole group of patients, and stratified by primary or SAI. Potential risk factors to be evaluated included DHEA replacement, glucocorticoid dose per body surface area, age at diagnosis, sex, educational status, prevalence of diabetes insipidus (in SAI), prevalence of concomitant disease, cause of AI (primary versus secondary), fludrocortisone dose (in PAI) and body mass index (BMI). We performed univariate and multivariate analyses, the latter including all of the above mentioned variables. All $P$ values are reported two-sided and considered statistically significant at a level $<0.05$. Analyses were performed using the statistical software package SPSS, version 17.0 (SPSS Inc., Chicago, IL, USA).

\section{Results}

\section{Patients}

From 883 patients with previously documented AI who were initially contacted, 526 sent an informed consent form back and received questionnaires. Sixty-two patients were subsequently excluded after review of the medical records because they did not fulfil the inclusion and exclusion criteria or due to insufficient data. Four hundred and forty-four patients were available for analysis. Data on age, duration of disease, underlying diagnosis and hormone replacement are given in Tables 1 and 2.

\section{Frequency of AC in primary and SAI}

In this survey, a total of $384 \mathrm{AC}$ in 6092 patient years were documented corresponding to an incidence rate of 6.3 crises per 100 patient years (6.6 in PAI and 5.8 in SAI). In terms of overall frequencies, at least one AC was reported by 185 of 444 patients $(41.7 \%)(119 / 254$ of patients with PAI $(46.9 \%)$ and $66 / 190$ of patients with SAI (34.9\%); Table 3). Ninetyfive $(21.4 \%)$ patients reported more than one AC (PAI $24 \%$ and SAI 18\%). Furthermore, need of i.v. glucocorticoid administration in an outpatient setting was reported by $108 / 254(42.5 \%)$ of the patients with PAI and by 54/190 (28.4\%) of the patients with SAI.

\section{Precipitating factors}

Precipitating factors are summarized in Table 4. Most important factors were infectious diseases, particularly gastrointestinal infections. Further causes were perioperative condition (PAI 7.2\% and SAI $15.5 \%$ of cases), exhaustive physical activity (PAI 7.7\% and SAI 7.3\% of cases), cessation of glucocorticoid replacement by the patient (PAI 5\% and SAI 6.4\% of cases) or the attending physician (PAI 1.1\% and SAI 3.6\%), psychic distress (PAI 3.3\% and SAI 3.6\%) and accidents (PAI 2.8\% and SAI $2.7 \%$ ). Also severe migraine, long distance flight, incident atrial fibrillation, climatic change with heat and grand mal epilepsy were reported as precipitating causes of AC. In a substantial proportion of cases, no underlying cause could be identified (PAI 6.6\% and SAI $12.7 \%$ of cases).

Table 2 Hormone replacement therapy in 444 patients with adrenal insufficiency (AI).

\begin{tabular}{|c|c|c|c|c|}
\hline \multirow[b]{2}{*}{$\begin{array}{l}\text { Primary adrenal } \\
\text { insufficiency }(n=254)\end{array}$} & \multicolumn{2}{|c|}{ Men $(n=63)$} & \multicolumn{2}{|c|}{ Women $(n=191)$} \\
\hline & $n$ & $\begin{array}{l}\text { Dose (mg/day) } \\
\text { median (range) }\end{array}$ & $n$ & $\begin{array}{l}\text { Dose (mg/day) } \\
\text { median (range) }\end{array}$ \\
\hline \multicolumn{5}{|c|}{ Al-specific replacement therapy } \\
\hline Hydrocortisone & 48 & $25(10-50)$ & 161 & $20(5-45)$ \\
\hline Cortisone acetate & 7 & $50(37.5-100)$ & 5 & $37.5(37.5-100)$ \\
\hline Prednisolone & 6 & $5(5-7.5)$ & 24 & $5(2-10)$ \\
\hline Dexamethasone $^{\mathrm{a}}$ & 4 & $0.25(0.25-0.5)$ & 7 & $0.25(0.25-1.5)$ \\
\hline Fludrocortisone & 56 & $0.1(0.05-0.2)$ & 173 & $0.1(0.03-0.5)$ \\
\hline \multirow[t]{2}{*}{ DHEA } & 3 & $25(25-25)$ & 61 & $25(12.5-25)$ \\
\hline & \multicolumn{2}{|c|}{ Men $(n=77)$} & \multicolumn{2}{|c|}{ Women $(n=113)$} \\
\hline $\begin{array}{l}\text { Secondary adrenal } \\
\text { insufficiency }(n=190)\end{array}$ & $n$ & $\begin{array}{l}\text { Dose (mg/day) } \\
\text { median (range) }\end{array}$ & $n$ & $\begin{array}{l}\text { Dose (mg/day) } \\
\text { median (range) }\end{array}$ \\
\hline \multicolumn{5}{|c|}{ Al-specific replacement therapy } \\
\hline Hydrocortisone & 55 & $20(10-50)$ & 89 & $20(5-35)$ \\
\hline Cortisone acetate & 2 & $25(25-25)$ & 5 & $37.5(31.5-43.7)$ \\
\hline Prednisolone & 20 & $5(2.5-7.5)$ & 17 & $5(2.5-7.5)$ \\
\hline Dexamethasone $^{a}$ & 0 & - & 1 & 0.25 \\
\hline DHEA & 3 & $25(25-25)$ & 4 & $25(12.5-25)$ \\
\hline
\end{tabular}

${ }^{a}$ Mostly in combination with hydrocortisone, prednisolone or cortisone acetate. 
Table 3 Frequency of adrenal crisis (AC) in patients with primary (PAI) and secondary adrenal insufficiency (SAI).

\begin{tabular}{lccrrr}
\hline & \multicolumn{2}{c}{ PAI $(n=254)$} & & \multicolumn{2}{c}{ SAI $(n=190)$} \\
\cline { 2 - 3 } \cline { 5 - 6 } Number of AC & \multicolumn{1}{c}{$n$} & $\%$ & & \multicolumn{1}{c}{$n$} & $\%$ \\
\hline 0 & 135 & 53.1 & & 124 & 65.1 \\
1 & 58 & 22.8 & & 32 & 16.9 \\
2 & 24 & 9.4 & & 8 & 4.2 \\
3 & 10 & 3.9 & & 12 & 6.3 \\
$\geq 4$ & 27 & 10.6 & & 14 & 7.4 \\
\hline
\end{tabular}

\section{Risk factors for AC}

Risk factor assessment was performed for the variables DHEA replacement, glucocorticoid dose per body surface area, age at diagnosis, sex, educational status, prevalence of diabetes insipidus (in SAI), prevalence of concomitant disease, cause of AI (primary versus secondary), fludrocortisone dose (in PAI) and BMI (Table 5).

PAI was associated with a higher incidence of AC in comparison to the patients with SAI (PAI: OR 0.51, 95\% CI $0.27-0.97, P=0.04)$. In PAI, patients with concomitant non-endocrine disease were at higher risk for $\mathrm{AC}(\mathrm{OR}=2.02,95 \% \mathrm{CI} 1.05-3.89, \mathrm{P}=0.04)$. In $\mathrm{SAI}$, female gender $(\mathrm{OR}=2.18,95 \%$ CI $1.06-4.5$, $P=0.04)$ and diabetes insipidus $(\mathrm{OR}=2.71,95 \% \mathrm{CI}$ $1.22-5.99, P=0.01)$ were significantly associated with the incidence of AC.

\section{Emergency equipment, patient education and dose adaptation}

An emergency card was carried by $93.8 \%$ of patients and $29.6 \%$ of patients were equipped with an emergency kit (glucocorticoid suppositories or glucocorticoid ampules for i.v. injection) respectively. On the other hand, $9.9 \%$ of patients reported that they never had increased their glucocorticoid dose.
Regarding patient education on crisis prevention and glucocorticoid dose adaptation, $15.6 \%$ felt 'very well' informed, $48.1 \%$ 'well' informed, $28.5 \%$ 'neither well nor badly' informed, $6.7 \%$ 'badly' informed and 1.1\% 'very badly' informed. This did not correlate with frequency of $\mathrm{AC}$ or emergency glucocorticoid administration.

\section{Discussion}

In this retrospective study, we have analyzed the epidemiology of AC in a large cohort of patients with primary and SAI. Adrenal crises occur in a substantial proportion of patients with AI with an overall frequency of $6.3 \mathrm{AC}$ per 100 patient years, which is higher than the frequency of $3.3 \mathrm{AC}$ per 100 patient years reported by Arlt et al. in 53 patients with PAI or SAI (14). Four or more adrenal crises were experienced by $7.4 \%$ of the patients with SAI and $10.6 \%$ of the patients with PAI. Similarly, in the international Addison's survey of the UK Addison's Disease Self-Help Group, 47\% of the participants reported that they had needed emergency treatment at a hospital since their diagnosis, and of these $10 \%$ reported that they had needed emergency hospital treatment on four or more occasions (17). Thus, AC is relatively common in patients with PAI or SAI with a frequency in the same order of magnitude as severe hypoglycaemia requiring assistance in patients with diabetes mellitus (about 10 per 100 patient years) $(18,19)$.

The main trigger for AC in our patient cohort was infectious diseases comprising $45 \%$ of all events. In a recent analysis of 1675 patients with PAI from Sweden, all-cause mortality was increased with a risk ratio of 2.19 for men and 2.86 for women (1). The risk ratio was highest for mortality from infectious disease ( 6.57 for men and 5.57 for women respectively), which emphasizes that patients with AI are at pronouncedly increased risk under conditions of infection. Slightly supraphysiological glucocorticoid replacement may

Table 4 Precipitating factors for adrenal crisis.

\begin{tabular}{|c|c|c|c|c|}
\hline & \multicolumn{2}{|r|}{ PAI } & \multicolumn{2}{|r|}{ SAI } \\
\hline & $n$ & Percentage of cases & $n$ & Percentage of cases \\
\hline Gastrointestinal infection & 59 & 32.6 & 24 & 21.8 \\
\hline Other infectious disease/fever & 44 & 24.3 & 19 & 17.3 \\
\hline Surgery & 13 & 7.2 & 17 & 15.5 \\
\hline Unknown & 12 & 6.6 & 14 & 12.7 \\
\hline Strenous physical activity & 14 & 7.7 & 8 & 7.3 \\
\hline Cessation of glucocorticoid substitution by patient & 9 & 5.0 & 7 & 6.4 \\
\hline Neglected glucocorticoid intake & 9 & 5.0 & 4 & 3.6 \\
\hline Psychic distress & 6 & 3.3 & 4 & 3.6 \\
\hline Accident & 5 & 2.8 & 3 & 2.7 \\
\hline Cessation of glucocorticoid substitution by attending physician & 2 & 1.1 & 4 & 3.6 \\
\hline Other reasons $^{\mathrm{a}}$ & 8 & 4.4 & 6 & 5.4 \\
\hline
\end{tabular}

Precipitating factors for adrenal crisis were reported for 181 cases in patients with primary adrenal insufficiency (PAI) and for 110 cases in patients with secondary adrenal insufficiency (SAI).

${ }^{a}$ Other reasons: primary adrenal insufficiency (PAI): e.g. migraine, after long distance flight, atrial fibrillation, insufficient dose adaptation during pregnancy; secondary adrenal insufficiency (SAI): e.g. difficulties with adjustment of substitution dose, climatic change (heat), grand mal epilepsy. 
Table 5 Risk factors for adrenal crisis.

\begin{tabular}{|c|c|c|c|}
\hline $\begin{array}{l}\text { Adrenal crisis with } \\
\text { hospital admission }\end{array}$ & $\begin{array}{l}\text { Odds } \\
\text { ratio }\end{array}$ & $\begin{array}{l}95 \% \\
\text { Confidence } \\
\text { interval }\end{array}$ & $\boldsymbol{P}$ \\
\hline \multicolumn{4}{|l|}{ All patients $(n=444)$} \\
\hline DHEA replacement & 0.88 & $0.52-1.49$ & 0.63 \\
\hline Glucocorticoid dose/BSA & 1.02 & $0.98-1.07$ & 0.32 \\
\hline Age at diagnosis & 0.98 & $0.96-1.01$ & 0.12 \\
\hline Female sex & 1.66 & $1.00-2.75$ & 0.05 \\
\hline Educational status & 1.22 & $0.70-2.14$ & 0.49 \\
\hline Concomitant disease & 1.81 & $1.13-2.90$ & 0.01 \\
\hline BMI & 0.98 & $0.93-1.03$ & 0.40 \\
\hline SAI versus PAI & 0.51 & $0.27-0.97$ & 0.04 \\
\hline \multicolumn{4}{|l|}{ Patients with PAI $(n=254)$} \\
\hline DHEA replacement & 0.82 & $0.40-1.72$ & 0.60 \\
\hline Glucocorticoid dose/BSA & 1.02 & $0.96-1.08$ & 0.50 \\
\hline Fludrocortisone dose & 0.84 & $0.38-1.88$ & 0.68 \\
\hline Age at diagnosis ${ }^{\mathrm{a}}$ & 0.96 & $0.93-0.99$ & 0.02 \\
\hline Female sex & 1.60 & $0.74-3.44$ & 0.23 \\
\hline Educational status & 0.90 & $0.37-2.19$ & 0.82 \\
\hline Concomitant disease & 2.02 & $1.05-3.89$ & 0.04 \\
\hline BMI & 0.995 & $0.91-1.08$ & 0.90 \\
\hline \multicolumn{4}{|l|}{ Patients with SAI $(n=189)$} \\
\hline DHEA replacement & 0.92 & $0.33-2.55$ & 0.87 \\
\hline Glucocorticoid dose/BSA & 1.04 & $0.96-1.12$ & 0.32 \\
\hline Age at diagnosis & 0.98 & $0.94-1.01$ & 0.21 \\
\hline Female sex & 2.18 & $1.06-4.50$ & 0.04 \\
\hline Diabetes insipidus & 2.71 & $1.22-5.99$ & 0.01 \\
\hline Educational status & 1.48 & $0.62-3.56$ & 0.38 \\
\hline Concomitant disease & 1.58 & $0.66-3.77$ & 0.31 \\
\hline BMI & 0.983 & $0.91-1.06$ & 0.67 \\
\hline
\end{tabular}

BSA, body surface area.

Bold indicates significant $P$ values $(P<0.05)$

${ }^{\mathrm{a}} \mathrm{Age}$ at diagnosis was negatively correlated with frequency of adrenal crisis.

predispose to infections (20), and inappropriate adaptation of glucocorticoid dose during infectious illness may further enhance this risk.

However, also severe migraine, long distance flight, incident atrial fibrillation, climatic change with heat, grand mal epilepsy or insufficient dose adaptation during pregnancy were reported as precipitating factors of AC. Particularly, climatic change with exposure to unusual heat was repeatedly reported as a condition that requires an increase of the replacement dose in PAI, probably also indicating the need of increased mineralocorticoid replacement. However, adjustment of mineralocorticoid dose is rarely considered by both patients and physicians.

Also psychic distress appears to be associated with a significantly increased demand of glucocorticoids. This was observed in $3.3 \%$ of the patients with PAI and $3.6 \%$ of the patients with SAI who reported that severe psychic distress had led to AC.

In many cases, the cause of $\mathrm{AC}$ remained unknown. This might in part be due to the retrospective nature of this survey. However, in our experience, AC sometimes develops rapidly without a clearly identifiable cause.

In an attempt to define risk factors for AC, Omori et al. (13) retrospectively analyzed the medical records of 22 patients with PAI and 115 patients with SAI. In this study, no difference in the frequency of $\mathrm{AC}$ between the patients with PAI and SAI was observed. However, patients with PAI may have been underrepresented. The authors identified mental retardation, age at diagnosis of $>20$ years and diabetes insipidus as risk factors for AC. In our analysis, we could not detect a relation to age at diagnosis or educational status. As most of our hypogonadal patients received replacement treatment for hypogonadism, we could not evaluate whether untreated hypogonadism represents a further risk factor as described by Omori et al. However, also in our cohort, diabetes insipidus was associated with an increased risk of AC in SAI patients. Thus, impaired vasopressin secretion may be an important pathophysiological factor. Replacement with desmopressin in diabetes insipidus activates V2 receptors only, and activation of V1 receptors may be missing during severe stress in these patients thereby precipitating AC. In a previous analysis, females with Addison's disease were at higher risk to develop AC (14). This is in concordance with our observations, also demonstrating a higher risk for AC in females with PAI. The reason for this female preponderance remains to be elucidated. Furthermore, patients with PAI experienced significantly more AC than patients with SAI, suggesting that lack of mineralocorticoids may represent a relevant additional risk factor.

Based on a postal survey in 97 patients from The Netherlands, Flemming et al. have demonstrated that $46 \%$ of patients were not sufficiently skilled in coping with stress, whereas $60 \%$ felt themselves to be well informed (21). In our cohort, $62.8 \%$ felt well or very well informed. However, no correlation with the frequency of AC could be detected, clearly indicating the need for improved prevention strategies. While diabetic patients and their partners undergo intensive and repeated structured education regarding prevention and handling of severe hypoglycaemia, patient education in AI is not yet well structured. Furthermore, evaluation of the effectiveness of patient education has never been performed. Although almost all our patients carried an emergency card, AC occurred in a substantial number of patients suggesting that current prevention strategies are not sufficient to protect from AC. Improved strategies may not only include a validated structured education of patients, but also more widespread provision with an emergency glucocorticoid set for i.v. administration and education of family members including training in the use of emergency glucocorticoids. Early aggressive treatment of infectious diseases is mandatory, and establishment of an emergency hotline for both patients and attending physicians should be considered to minimize hospitalization for $\mathrm{AC}$ and to completely eliminate death from $\mathrm{AC}$ in patients with known chronic AI. The efficacy of such measures has to be evaluated in prospective studies.

Our study has several limitations: first, it is a retrospective investigation in patients suffering from adrenal disease for up to 57 years. Thus, it might have been difficult to exactly remember the number, 
precipitating factors and circumstances of every AC. Furthermore, it is difficult to judge in all cases whether true AC was present. Hydrocortisone may have been administered intravenously as pre-emptive action to avert clinical aggravation. In many cases, gastrointestinal infection was indicated as cause of AC. However, it is often unclear whether a gastrointestinal infection was actually the underlying cause or whether diarrhoea and vomiting were already manifestations of AC. There might be some risk of overestimation of incidence of $\mathrm{AC}$ as patients from the self-help group were included and patients that have no or only minor complaints might not have participated. However, in our cohort, $40 \%$ of the patients from the self-help group experienced at least one AC compared to $42 \%$ of the patients from the University Hospitals Berlin and Würzburg.

Furthermore, incidence, causes and risk factors of AC were only one aspect of the survey that also evaluated further aspects of the adrenal disease (e.g. subjective health status, replacement regimens, problems prior diagnosis of AI, etc.) $(15,16,22)$.

The exact incidence and causes of $\mathrm{AC}$ have to be addressed in a prospective trial.

In conclusion, AC occurs in a substantial proportion of both PAI and SAI patients, mainly triggered by infectious disease. However, also several other causes need to be taken into account for adjustments of the glucocorticoid dose. The regular occurrence of life-threatening $\mathrm{AC}$ in seemingly well-educated patients clearly indicates the need for improved prevention strategies.

\section{Declaration of interest}

The authors declare that there is no conflict of interest that could be perceived as prejudicing the impartiality of the research reported.

\section{Funding}

This work was supported by the Wilhelm-Sander-Stiftung (project grants 2003.175.1 and 2003.175.2 to B Allolio and S Hahner).

\section{Acknowledgements}

We are indebted to Sven Diederich, Reinhard Finke and Ullrich Bogner, all Berlin, for recruitment of patients. We thank Prof. J Hensen, Prof. C Schöfl, Mrs Hummel, Mrs Jalowski and Mrs Stahl from the German Self-Help Network for Pituitary and Adrenal Diseases 'Netzwerk Hypophysen- und Nebennierenerkrankungen e. V.' for their invaluable support by contacting the patients of this network and distributing the questionnaires.

\section{References}

1 Bergthorsdottir R, Leonsson-Zachrisson M, Oden A \& Johannsson G. Premature mortality in patients with Addison's disease: a population-based study. Journal of Clinical Endocrinology and Metabolism 200691 4849-4853.

2 Erichsen MM, Lovas K, Fougner KJ, Svartberg J, Hauge ER, Bollerslev J, Berg JP, Mella B \& Husebye ES. Normal overall mortality rate in Addison's disease, but young patients are at risk of premature death. European Journal of Endocrinology 2009160 233-237.
3 Shulman DI, Palmert MR \& Kemp SF. Adrenal insufficiency: still a cause of morbidity and death in childhood. Pediatrics 2007119 e484-e494.

4 Graves L III, Klein RM \& Walling AD. Addisonian crisis precipitated by thyroxine therapy: a complication of type 2 autoimmune polyglandular syndrome. Southern Medical Journal 200396 824-827.

5 Davis J \& Sheppard M. Acute adrenal crisis precipitated by thyroxine. BMJ 19862921595.

6 Fonseca V, Brown R, Hochhauser D, Ginsburg J \& Havard CW. Acute adrenal crisis precipitated by thyroxine. BMJ 1986292 1185-1186.

7 Lundy JB, Slane ML \& Frizzi JD. Acute adrenal insufficiency after a single dose of etomidate. Journal of Intensive Care Medicine 200722 111-117.

8 Sarver RG, Dalkin BL \& Ahmann FR. Ketoconazole-induced adrenal crisis in a patient with metastatic prostatic adenocarcinoma: case report and review of the literature. Urology 199749 781-785.

9 Khosla S, Wolfson JS, Demerjian Z \& Godine JE. Adrenal crisis in the setting of high-dose ketoconazole therapy. Archives of Internal Medicine 1989149 802-804.

10 Braatvedt GD, Newrick PG \& Corrall RJ. Patients' self administration of hydrocortisone. BMJ 19903011312.

11 Nagesser SK, van Seters AP, Kievit J, Hermans J, Krans HM \& van de Velde CJ. Long-term results of total adrenalectomy for Cushing's disease. World Journal of Surgery 200024 108-113.

12 Thompson SK, Hayman AV, Ludlam WH, Deveney CW, Loriaux DL \& Sheppard BC. Improved quality of life after bilateral laparoscopic adrenalectomy for Cushing's disease: a 10-year experience. Annals of Surgery 2007245 790-794.

13 Omori K, Nomura K, Shimizu S, Omori N \& Takano K. Risk factors for adrenal crisis in patients with adrenal insufficiency. Endocrine Journal 200350 745-752.

14 Arlt W \& Allolio B. Adrenal insufficiency. Lancet $2003 \mathbf{3 6 1}$ 1881-1893.

15 Hahner S, Loeffler M, Fassnacht M, Weismann D, Koschker AC, Quinkler M, Decker O, Arlt W \& Allolio B. Impaired subjective health status in 256 patients with adrenal insufficiency on standard therapy based on cross-sectional analysis. Journal of Clinical Endocrinology and Metabolism 200792 3912-3922.

16 Bleicken B, Hahner S, Loeffler M, Ventz M, Allolio B \& Quinkler M. Impaired subjective health status in chronic adrenal insufficiency: impact of different glucocorticoid replacement regimens. European Journal of Endocrinology 2008159 811-817.

17 White K \& Arlt W. Adrenal crisis in treated Addison's disease: a predictable but under-managed event. European Journal of Endocrinology 2009162 115-120.

18 Shalitin S \& Phillip M. Hypoglycemia in type 1 diabetes: a still unresolved problem in the era of insulin analogs and pump therapy. Diabetes Care 200831 (Suppl 2) S121-S124.

19 Swinnen SG, Mullins P, Miller M, Hoekstra JB \& Holleman F. Changing the glucose cut-off values that define hypoglycaemia has a major effect on reported frequencies of hypoglycaemia. Diabetologia 200952 38-41.

20 Saag KG, Koehnke R, Caldwell JR, Brasington R, Burmeister LF, Zimmerman B, Kohler JA \& Furst DE. Low dose long-term corticosteroid therapy in rheumatoid arthritis: an analysis of serious adverse events. American Journal of Medicine 199496 115-123.

21 Flemming TG \& Kristensen LO. Quality of self-care in patients on replacement therapy with hydrocortisone. Journal of Internal Medicine 1999246 497-501.

22 Bleicken B, Hahner S, Loeffler M, Ventz M, Decker O, Allolio B \& Quinkler M. Influence of hydrocortisone dosage scheme on health related quality of life in patients with adrenal insufficiency. Clinical Endocrinology 2009. In Press DOI: 10.1111/ j.1365-2265.2009.03596.x.

Received 18 November 2009

Accepted 23 November 2009 